SN: 2641-1644

\title{
Nonmosaic Klinefelter Syndrome Successful Conception after TESE/ICSI: A case report
}

\section{SayedElAkhras ${ }^{1}$, Karim Omar ElSaeed ${ }^{2}$, Mohamed Halawa $^{3}$, M Yousef Elsemary ${ }^{4}$, AlyElakhras ${ }^{5}$, Gajek Adam ${ }^{6}$ and Vasilios Tanos*7}

${ }^{1}$ Department of Gynecology and Obstetrics, Omam Hospital, Cairo, Egypt

${ }^{2}$ Department of Urology, Ain Shams University, Cairo, Egypt

${ }^{3,5}$ Department of Embryology, Omam Hospital, Cairo, Egypt

${ }^{4}$ Department of Gynaecology and Obstetrics, ElGalaa Maternity Teaching Hospital, Cairo, Egypt

${ }^{6}$ Medical School, St Georges University of London, Cyprus

${ }^{7}$ Nicosia University, Director Research and Development Omam Hospital, Egypt

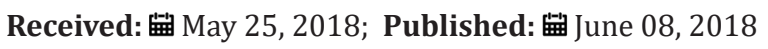

*Corresponding author: Vasilios Tanos, Nicosia University Medical School, and Aretaeio Hospital, Nicosia, Cyprus and Director Research and Development Omam Hospital, Cairo, Egypt

\begin{abstract}
Klinefelter syndrome patients are mostly clinically azoospermic, and before the era of TESE, were unable to father genetically their own offspring. Nonmosaic Klinefelter [NMK] patients have chances of fatherhood once sperm is harvested from the epididymal seminiferous tubules and further injected in the ovarian cytoplasm. We report a case of 36y old NMK patient, treated for 3 months with supportive and hormonal medication achieving a pregnancy after TESE/ICSI. This is the first published case of successful conception in a couple with a nonmosaic Klinefelter father in Egypt using TESE/ICSI. Review of the literature for any advantages of mTESE over TESE and conception demonstrated higher pregnancy rates by TESE although the sperm retrieval rate was similar in both techniques.
\end{abstract}

Conclusion: Nonmosaic Klinefelter patients should no longer be considered sterile.

Keywords: Klinefelter Syndrome; Azoospermia; Male Infertility; TESE; ICSI

\section{Introduction}

Klinefelter syndrome [KS] was first described in 1942 by Harry Klinefelter [1]. It is the most common chromosomal disorder in men. The estimated prevalence of $\mathrm{K}$ Sis $0.2 \%$ in the general population, $3 \%$ among infertile men, and up to $11 \%$ in men with non-obstructive azoospermia [2]. Yet, KS remains frequently under diagnosed, because of the wide phenotypic variation among affected individuals, and the lack of established screening programs. Only $25 \%$ of men with KS are diagnosed during their lifetime, with fewer than $10 \%$ being diagnosed before puberty [3]. The disorder is categorized by $\mathrm{X}$-chromosome polysomy, with X-disomy [47, $\mathrm{XXY}$ ] being the most common variant representing about $85 \%$ $95 \%$ of the cases. Characteristic features of KS include small testes, hypogonadism, and infertility [4]. Higher grades of X chromosome polysomy are associated with a more severe clinical presentation, whereas genetic mosaicism (46, XY/47, XXY) usually results in a milder phenotype [5].

Since the introduction of intra-cytoplasmic sperm injection [ICSI] [6] and testicular sperm extraction [TESE] [7], a considerable number of men with Klinefelter syndrome have been able to father genetically own offspring. Looking at the three of the more recent published studies, in 2015 Ozveri et al. [8] confirms that sperm retrieval is possible in azoospermatic Klinefelter Syndrome patients, and recommends the use of artificial reproductive techniques (ART) for those wishing to conceive. In 2016 Ishikawa et al. [9] concluded that the use of mTESE to extract motile sperm is vital in the use of Klinefelter Syndrome cases in order to maximize the chances of 
achieving a clinical pregnancy and embryological development. In 2016 Vicdan et al. [10] concluded that with regards to the use of fresh vs. cryopreserved sperm in ICSI cycles demonstrated an equal chance of success. In this case report a successful conception in an azoospermic nonmosaic Klinefelter father using a combined TESEICSI protocol is highlighted.

\section{Case Presentation}

A 36 year old Egyptian male of rural origin presented to our fertility clinic complaining of primary infertility for 9 years. He enjoyed a good sex life, without any concern regarding libido, erection, intercourse and ejaculation. His wife was 10 years younger and her menstrual history was unremarkable. General examination diagnoses well developed secondary male sexual characteristics, $187 \mathrm{~cm}$ tall and weight of $82 \mathrm{Kg}$. Genital examination revealed an average sized penis with normal male pubic hair pattern and two small firm testicles. Both vas were palpable and showed no abnormality. Repeated spermo grams in 3 months intervals and 3 days of abstinence diagnose total azoospermia. The second result was a duplicate of the first one and the results of the hormonal assay showed a hypergonadotrophic hypogonadism state (Table 1). Karyotyping followed clinical data, raised suspicion of Klinefelter Syndrome, reporting a nonmosaic Klinefelter (Figure 1).

Table 1: The hormonal assay of our patient.

\begin{tabular}{|c|c|c|}
\hline Hormone & Result & Reference Range \\
\hline FSH $^{*}$ & $25.82 \mathrm{mIU} / \mathrm{mL}$ & $1.5-12.4$ \\
\hline LH$^{*}$ & $21.78 \mathrm{mIU} / \mathrm{mL}$ & $1-9.3$ \\
\hline Testosterone & $191 \mathrm{ng} / \mathrm{dL}$ & $300-1000$ \\
\hline Prolactin & $5.85 \mathrm{ng} / \mathrm{mL}$ & $2.58-21.4$ \\
\hline Estradiol $[\mathrm{E} 2]$ & $34.5 \mathrm{pg} / \mathrm{mL}$ & $7.63-52$ \\
\hline AMH* & $0.23 \mathrm{ng} / \mathrm{mL}$ & $1.5-10.6$ \\
\hline TSH $^{*}$ & $1.08 \mathrm{mIU} / \mathrm{mL}$ & $0.27-4.2$ \\
\hline
\end{tabular}

*FSH=Follicle Stimulating Hormone, LH=Luteinizing Hormone, AMH=Anti-Mullerian Hormone, TSH=Thyroid Stimulating Hormone.

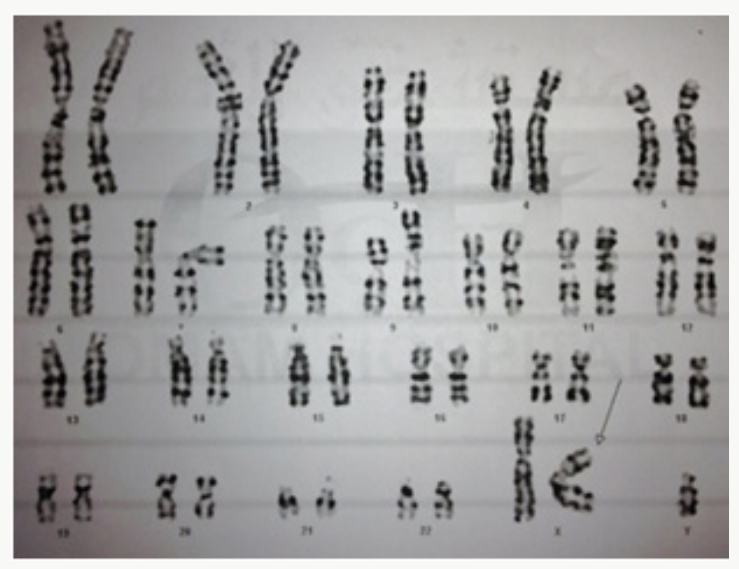

Figure 1: Karyotyping of the patient. Notice the arrow pointing out the extra $\mathrm{X}$ chromosome.
Although the value of treatment of cases with hypergonadotrophic hypogonadism is controversial, the patient was treated for 3 months with combined hormonal and nonhormonal supplement therapy prior to TESE (Table 2). An attempt to enhance the harvest of the scheduled testicular sperm extraction and improving ICSI chances of fertilization. TESE was performed and multiple sections were retrieved from both testes. Primary scanning of the surgical harvest revealed azoospermia. After processing, the left testis showed motile sperms from 2 tissue cores, allowing for ICSI as well as a cryopreservation vial for future interest. The operation was uneventful. Postoperatively mild left orchitis was diagnosed which subsided after a 10day course of quinolones, anti-inflammatory drugs and scrotal elevation.

Table 2: Details of our 3 month regimen prescribed to our patient prior to TESE.

\begin{tabular}{|c|c|}
\hline Drug & Dose \\
\hline Follitropinalfa & 75 IU subcutaneous 3 times weekly \\
\hline hCG* & 500 IU subcutaneous 3 times weekly \\
\hline Vitamin E & 400 IU per day \\
\hline Relora & 250 mg per day \\
\hline Vitamin C & 200 mg per day \\
\hline Lycopene & 100 mg per day \\
\hline Zinc & 70 mg per day \\
\hline L-Methionine & 25 mg per day \\
\hline Vitamin A & 5000 IU per day \\
\hline Folic acid & 1000 mcg per day \\
\hline Selenium & 250 mcg per day \\
\hline Vitamin B12 & 15 mcg per day \\
\hline
\end{tabular}

The wife was treated with long GnRH agonist regimen, pituitary down-regulation with Triptorelin $0.1 \mathrm{mg}$ once daily SC injection [decapeptyl;Ferring], commenced in the midluteal phase of the menstrual cycle and continued for 2 weeks. This was followed by estradiol blood level and Trans vaginal ultrasound confirmation of down-regulation, recording the leading follicle diameter and antral follicular count $[\mathrm{AFC}]$. Human menotropin, gonadotropin injections at a dose of 225IU/day [menogon;Ferring] initiated and continued until leading follicle reach $24 \mathrm{~mm}$ maximum diameter, within high range estradiol level and low progesterone level. The hCG injection [Choriomon 5000 IU/day; IBSA; Egypt] was administered when at least three follicles reach $\mathrm{R} 17 \mathrm{~mm}$ in diameter. Vaginal egg collection was scheduled 36 hours following hCG triggering. TESE was performed before oocyte retrieval in order to confirm a positive retrieval of sperms. Microscopy of the retrieved semen isolated 28 out of total 58 spermatocytes. The rest were either immotile or with sluggish tail movement. Overall 22 oocytes were retrieved while 15 diagnosed at MII stage. ICSI was performed and 6 good 
quality A and B embryos progressed to day 3. Three embryos at stage A were transferred on day D3. Twelve days post ET the beta hCG was $141.0 \mathrm{mIU} \backslash \mathrm{m}$. The lady delivered at term, a healthy female baby of $3000 \mathrm{gr}$.

\section{Discussion}

Historically, men with KS were considered infertile. However, it is now well accepted that isolated foci of spermatogenesis can exist in the testes of patients with KS [11]. This discovery, along with advances in assisted reproductive technologies [ART] during the past two decades, has made paternity possible for men with KS. Surgical sperm retrieval and intracytoplasmic sperm injection [ICSI] have dramatically improved the fertility potential of men with KS. Tournaye et al. first reported on successful sperm retrieval in men with KS using TESE in 1996 [12]. The first pregnancies achieved using ICSI of ejaculated and testicular sperm were reported 2 years later [13]. Since then, there have been reported 101 children born to fathers with nonmosaic KS [14].

With the use of micro dissection TESE, sperm retrieval rates in patients with KS are considered equivalent to those in men with non-obstructive azoospermia. The outcome of mTESE versus TESE in non-obstructive azoospermia was also investigated by a systematic review published in 2014. Seven out of 62 identified studies were included in the final analysis. SRR was significantly higher in the mTESE group (42.9-63\%) in comparison with TESE (16.7-45\%) [15]. In another retrospective cohort study among 714 men with NOA, $40.5 \%$ had successful sperm retrieval at their first TESE. In total, 261 couples had 444 ICSI cycles and 48 frozen embryo transfer cycles, leading to 129 pregnancies and 96 live birth deliveries. The expected cumulative delivery rates after six ICSI cycles were 78\%.Authors indicated the intrinsic limitations related to the retrospective cohort study design [16]. Our recent published, review results demonstrate among 1,070 KS patients treated by ICSI the SRR was $46.3 \%$ and PR was $21 \%$ with mTESE and $45.6 \%$ SRR and $44.4 \%$ PR with TESE [17]. Fewer complications were observed on ultrasound examination after mTESE procedure [15].

Mehta A et al. [18] published a systematic review/metaanalysis to investigate the effect of early hormonal therapy [HR] on sperm retrieval rates in patients with Klinefelter Syndrome [KS]. Although the studies varied in their conclusions as to predictors of sperm retrieval, positive predictors included young age and preoperative Testosterone levels close to or within the normal range, either at baseline or with hormonal therapy [aromatase inhibitors, clomiphene citrate [CC], or hCG] [19-22] Several authors have identified "young" patient age, usually defined as age $<30$ years, as being the most consistent positive predictor of sperm retrieval in patients with KS $[23,24]$. The age of the reported patient was 36 years who does not fit with most the studies age predictor for successful retrieval. However, his testosterone level was low and the treatment before surgery might have had a positive impact upon successful sperm retrieval.

\section{Conclusion}

The majority of 47, XXY males are azoospermic and considered sterile until the introduction of TESE/ICSI. Solid parameters to distinguish patients with KS and fertilization potential and chance of fatherhood are missing. A prospective randomized control study would probably reveal those important parameters indicating KS patients with higher chance to achieve a pregnancy.

\section{References}

1. HF Klinefelter, EC Reifenstein, F Albright (1942) Syndrome characterized by gynecomastia, aspermatogenesis without a-leydigism, and increased excretion of follicle-stimulating hormone. J Clin Endocrinol 2(11): 615627.

2. C Foresta, C Galeazzi, A Bettella, Marin P, Rossato M, et al. (1999) Analysis of meiosis in intratesticular germ cells from subjects affected by classic Klinefelter's syndrome. J Clin Endocrinol Metab 84(10): 3807-3810.

3. A Bojesen, S Juul, CH Gravholt (2003) Prenatal and postnatal prevalence of Klinefelter syndrome: a national registry study. J ClinEndocrinolMetab 88(2): 622-626.

4. M Ando, K Yamaguchi, K Chiba, Miyake H, Fujisawa M (2013) Outcome of microdissection testicular sperm extraction in azoospermic patients with Klinefelter syndrome and other sex-chromosomal anomalies. SystBiolReprodMed 59(4): 210-213.

5. R Sarkar, KM Marimuthu (1983) Association between the degree of mosaicism and the severity of syndrome in Turner mosaics and Klinefelter mosaics. Clin Genet 24(6): 420-428.

6. G Palermo, H Joris, P Devroey (1992) Pregnancies after intracytoplasmic injection of single spermatozoon into an oocyte. Lancet 340(8810): 1718.

7. SJ Silber, AC Van Steirteghem, J Liu, Nagy Z, Tournaye H, et al. (1995) High fertilization and pregnancy rate after intracytoplasmic sperm injection with spermatozoa obtained from testicle biopsy. Hum Reprod 10(1): 148-152.

8. H Ozveri, F Kayabasoglu, C Demirel (2015) Outcomes of micro-dissection TESE in patients with non-mosaic Klinefelter's syndrome without hormonal treatment. Int J FertilSteril 8(4): 421-428.

9. T Ishikawa, S Mizuta, K Yamaguchi, Y Takaya, R Nishiyama, et al. (2016) Clinical outcomes of microdissection testicular sperm extraction [micro TESE] and intracytoplasmic sperm injection [ICSI] in patients with Klinefelter syndrome-Fertility and Sterility 106(3): e298.

10. K Vicdan, C Akarsu, Sozen, Buluç B, Vicdan A, et al. (2016) Outcome of intracytoplasmic sperm injection using fresh and cryopreservedthawed testicular spermatozoa in 83 azoospermic men with Klinefelter syndrome. Journal of Obstetrics and Gynaecology Research 42(11): 1558-1566.

11. PN Schlegel (2009) Nonobstructive azoospermia: a revolutionary surgical approach and results. SeminReprodMed 27(2): 165-170.

12. H Tournaye, C Staessen, I Liebaers, Van Assche E, Devroey P, et al. (1996) Testicular sperm recovery in nine 47, XXY Klinefelter patients. Hum Reprod 11(8): 1644-1649.

13. GD Palermo, PN Schlegel, ES Sills, Veeck LL, Zaninovic N, et al. (1998) Births after intracytoplasmic injection of sperm obtained by testicular extraction from men with nonmosaic Klinefelter's syndrome. N Engl J Med 338(9): 588-590.

14. G Fullerton, M Hamilton, A Maheshwari (2010) Should non-mosaic Klinefelter syndrome men be labeled as infertile in 2009.Hum Reprod 25(3): 588-597. 
15. Y Deruyver, D Vanderschueren, F Van der Aa (2014) Outcome of microdissection TESE compared with conventional TESE in nonobstructive azoospermia. A systematic review. Andrology 2(1): 20-24.

16. V Vloeberghs, G Verheyen, P Haentjens, A Goossens, NP Polyzos, et al. (2015) How successful is TESE-ICSI in couples with non-obstructive azoospermia. Hum Reprod 30(8): 1790-1796.

17. V Tanos, A Gajek, MY Elsemary, KO ElSaeed, KE Berry, et al. (2018) Klinefelter Syndrome: Review of the Literature Comparing TESE and mTESE. Sperm Retrieval and Pregnancy Rate. Int J Reprod Med Gynecol 4(1): 012-016.

18. A Mehta, DA Paduch (2012) Klinefelter syndrome: an argument for early aggressive hormonal and fertility management. FertiSteril 98(2): 274283.

19. K Kyono, H Uto, Y Nakajo, S Kumagai, Yasuhisa Araki, et al. (2007) Seven pregnancies and deliveries from non-mosaic Klinefelter syndrome patients using fresh and frozen testicular sperm. J Assist Reprod Genet 24(1): 47-51.
20. H Okada, K Goda, Y Yamamoto, Sofikitis N, Miyagawa I, et al. (2005) Age as a limiting factor for successful sperm retrieval in patients with nonmosaic Klinefelter's syndrome. FertilSteril 84(6): 1662-1664.

21. I Madgar, J Dor, R Weissenberg, Gil Raviv, Jacob Levron, et al. (2002) Prognostic value of the clinical and laboratory evaluation in patients with nonmosaic Klinefelter syndrome who are receiving assisted reproductive therapy. FertilSteril 77(6): 1167-1169.

22. R Ramasamy, JA Ricci, GD Palermo, Gosden LV, Rosenwaks Z, et al. (2009) Successful fertility treatment for Klinefelter's syndrome. J Urol 182(3): 1108-1113.

23. M Emre Bakircioglu, HF Erden, T Kaplancan, Ciray N, Bener F, et al. (2006) Aging may adversely affect testicular sperm recovery in patients with Klinefelter syndrome. Urology 68(5): 1082-1086.

24. H Okada, K Goda, Y Yamamoto, Sofikitis N, Miyagawa I, et al. (2005) Age as a limiting factor for successful sperm retrieval in patients with nonmosaicKlinefelter's syndrome. Fertil Steril 84(6): 1662-1664.

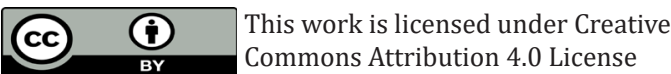

Submission Link:

Submit Article

DOI: $10.32474 /$ OAJRSD.2018.01.000107

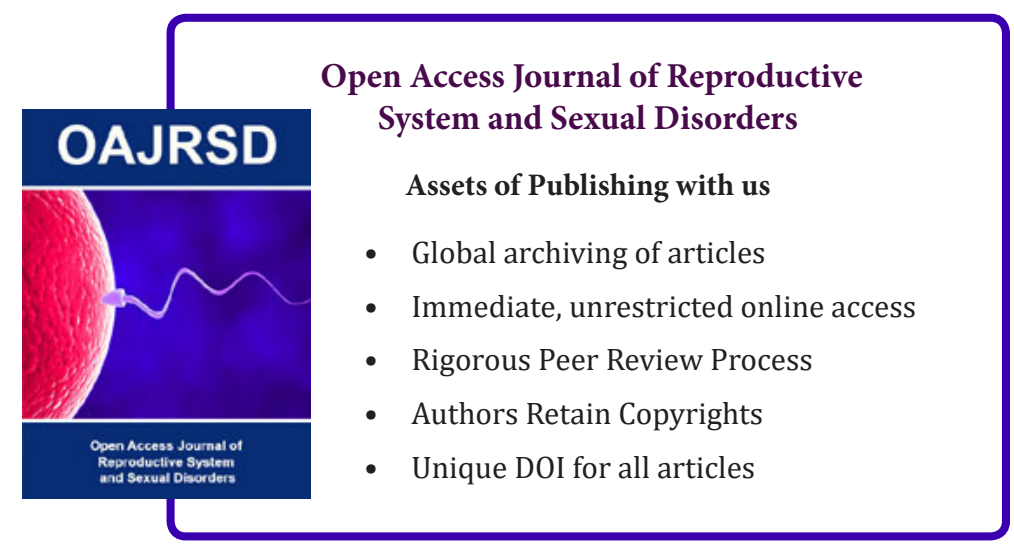

